\title{
Benchmarks for Higgs Pair Production and Heavy Higgs Searches in the Two-Higgs-Doublet Model of Type II
}

\author{
Julien Bagliq* \\ Institut für Theoretische Physik, Karlsruhe Institute of Technology, \\ Engesserstraße 7, D-76128 Karlsruhe, Germany \\ Otto Eberhardt $\dagger^{\dagger}$ \\ Istituto Nazionale di Fisica Nucleare, Sezione di Roma, \\ Piazzale Aldo Moro 2, I-00185 Roma, Italy \\ Ulrich Nierste \\ Institut für Theoretische Teilchenphysik, Karlsruhe Institute of Technology, \\ Engesserstraße 7, D-76128 Karlsruhe, Germany
}

Martin Wiebusch§

Institute for Particle Physics and Phenomenology, Durham University, Durham DH1 3LE, United Kingdom

(Dated: May 28, 2014)

The search for additional Higgs particles and the exact measurements of Higgs (self-) couplings is a major goal of future collider experiments. In this paper we investigate the possible sizes of new physics signals in these searches in the context of the $C P$-conserving two-Higgs doublet model of type II. Using current constraints from flavour, electroweak precision, and Higgs signal strength data, we determine the allowed sizes of the triple Higgs couplings and the branching fractions of the heavy Higgs bosons into lighter Higgs bosons. Identifying the observed Higgs resonance with the light $C P$-even 2HDM Higgs boson $h$, we find that the $h h h$ coupling cannot exceed its SM value, but can be reduced by a factor of 0.56 at the $2 \sigma$ level. The branching fractions of the heavy neutral Higgs bosons $H$ and $A$ into two-fermion or two-vector-boson final states can be reduced by factors of 0.4 and 0.01 , respectively, if decays into lighter Higgs boson are possible and if the mass of the decaying Higgs is below the $t \bar{t}$ threshold. To facilitate future studies of collider signatures in 2HDM scenarios with large triple Higgs couplings or decay modes of the heavy Higgs bosons not covered by the SM Higgs searches we provide a set of benchmark points which exhibit these features and agree with all current constraints. We also discuss the effect of the heavy Higgs bosons on the $g g \rightarrow h h$ cross section at a $14 \mathrm{TeV}$ LHC for some of these benchmarks. For $m_{H}$ below the $h h$ threshold we see a reduction of the SM $g g \rightarrow h h$ cross section due to destructive interference, but for $m_{H}$ above the $h h$ threshold current constraints allow enhancement factors above 50. An enhancement factor of 6 is still possible in scenarios in which the heavy Higgs particles would not be discovered by standard searches after $300 \mathrm{fb}^{-1}$ of data.

\section{INTRODUCTION}

The discovery of a Higgs boson with a mass of $126 \mathrm{GeV}$ is the undisputed highlight of Run I

* julien.baglio@kit.edu

† otto.eberhardt@roma1.infn.it

$\ddagger$ ulrich.nierste@kit.edu

$\S$ martin.wiebusch@durham.ac.uk of the Large Hadron Collider (LHC) at CERN [1, 2]. The measured signal strengths agree with the Standard Model (SM) predictions in several production and decay channels of the discovered 


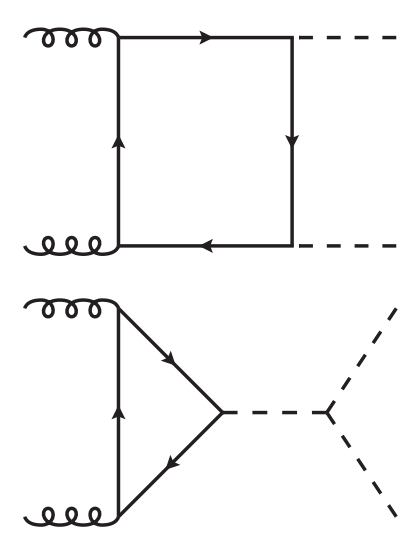

FIG. 1. Representative diagrams contributing to $g g \rightarrow h h$. Other diagrams are obtained by permuting the external lines. In the 2HDM both $h$ and $H$ can be exchanged between the top loop and the $h$ pair in the right diagram.

particle [3, 4, establishing the Higgs mechanism [5] -7 as the correct theoretical framework of elementary particle mass generation. This achievement has been recognised with the 2013 Nobel Prize for Physics for Peter Higgs and François Englert. However, it is not clear at all whether the Higgs sector is indeed minimal, containing only a single Higgs doublet. The most straightforward way to go beyond the SM is the addition of a second Higgs doublet [8] to the field content of the model. In this paper we focus on the $C P$-conserving two-Higgs-doublet-model (2HDM) of type II and identify the observed $126 \mathrm{GeV}$ state as the lightest $C P$-even Higgs boson $h$. Currently a lot of effort is spent to confront the above-described model to LHC data [930. Remarkably, current data do not at all push the model parameters to the decoupling limit $m_{A, H, H^{ \pm}} \rightarrow \infty$, but permit $A$ and $H$ masses well below $200 \mathrm{GeV}$ [29, 31 33 .

A major target of the future LHC programme is the search for Higgs pair production. In the gluon fusion channel, which is the dominant production process, there are two classes of diagrams at leading order contributing to this process, depicted in Fig. 1. Higgs pair production probes the triple- $h$ coupling which in the SM is entirely fixed in terms of the Higgs mass and vacuum expectation value. The corresponding diagram (Fig. 1b) is smaller than the box dia- gram by roughly a factor of 3 , depending on the invariant mass of the Higgs pair.

In models with extended Higgs sectors the value of the triple- $h$ coupling can differ from its SM value and additional Higgs bosons can be exchanged in the $s$-channel. Higgs pair production at hadron and linear colliders has been studied extensively in the literature [34 41], and QCD corrections to the SM $g g \rightarrow h h$ cross section, which is the dominant process, are known to next-to-leading order in the infinite top mass approximation [42, to subleading orders in $1 / m_{t}$ [43] and beyond next-to-leading order [44 46]. Parton-shower effects have been studied in [47. A recent study [48] has found that within the SM the triple- $h$ coupling can be measured with an accuracy of $40 \%$ at the LHC with an integrated luminosity of $3 \mathrm{ab}^{-1}$ collected at an energy of $14 \mathrm{TeV}$. Other studies have addressed extended Higgs sectors [49, 50] and the sensitivity of the triple Higgs coupling to new physics [51].

The purpose of this paper is to use all current constraints on the type-II 2HDM parameter space to determine the allowed ranges of the triple-Higgs couplings and the branching fractions of the heavy Higgs bosons in order to prepare the ground for collider studies of the various Higgs pair production and decay channels. Specifically, we address the following questions:

i) To what extent can the triple- $h$ coupling deviate from its SM value?

ii) What are the maximal sizes of the tripleHiggs couplings involving $H, A$ or $H^{ \pm}$?

iii) Can decays like $H \rightarrow h h$ or $A \rightarrow Z h$ lead to a large suppression of the branching fractions in the standard searches for $H$ and $A$ ?

iv) To what extent can $g g \rightarrow h h$ be enhanced with respect to the SM prediction?

To facilitate future detailed studies of collider signatures we provide a set of benchmark points which maximise the above-mentioned features, but still agree with all experimental and theoretical constraints.

This paper is organised as follows: in Section II we recall the considered model and fix 
our notations. Section III discusses the input to our analysis, namely Higgs signal strengths, electroweak precision observables, and relevant flavour observables. Section [V] addresses questions i) and ii) above. Questions iii) and iv) are discussed in Section V, where we define the benchmark scenarios. In Section VI we present our conclusions.

\section{THE TWO-HIGGS-DOUBLET MODEL OF TYPE II}

In this paper we consider the $C P$-conserving two-Higgs-doublet-model of type II with a softly broken $Z_{2}$ symmetry. For details about this model we refer to [52, whose notations we adopt here. The Higgs potential is given by

$$
\begin{aligned}
V= & m_{11}^{2} \Phi_{1}^{\dagger} \Phi_{1}+m_{22}^{2} \Phi_{2}^{\dagger} \Phi_{2}-m_{12}^{2}\left(\Phi_{1}^{\dagger} \Phi_{2}+\Phi_{2}^{\dagger} \Phi_{1}\right) \\
& +\frac{1}{2} \lambda_{1}\left(\Phi_{1}^{\dagger} \Phi_{1}\right)^{2}+\frac{1}{2} \lambda_{2}\left(\Phi_{2}^{\dagger} \Phi_{2}\right)^{2} \\
& +\lambda_{3}\left(\Phi_{1}^{\dagger} \Phi_{1}\right)\left(\Phi_{2}^{\dagger} \Phi_{2}\right)+\lambda_{4}\left(\Phi_{1}^{\dagger} \Phi_{2}\right)\left(\Phi_{2}^{\dagger} \Phi_{1}\right) \\
& +\frac{1}{2} \lambda_{5}\left[\left(\Phi_{1}^{\dagger} \Phi_{2}\right)^{2}+\left(\Phi_{2}^{\dagger} \Phi_{1}\right)^{2}\right] .
\end{aligned}
$$

The physical scalar spectrum of this model consists of two $C P$-even neutral scalars $h$ and $H$, a $C P$-odd neutral scalar $A$ and a charged scalar $H^{ \pm}$. The masses of these states are denoted as $m_{\phi}$ with $\phi \in h, H, A, H^{ \pm}$. Throughout this paper we assume that the light $C P$ even scalar $h$ is the observed Higgs resonance and keep $m_{h}=$ $126 \mathrm{GeV}$ fixed. For the remaining independent real parameters of the model we choose

$$
\begin{aligned}
& \tan \beta=v_{2} / v_{1}, \beta-\alpha, m_{12}^{2}, \\
& m_{H}, m_{A}, m_{H^{ \pm}}
\end{aligned}
$$

where $v_{1} / \sqrt{2}$ and $v_{2} / \sqrt{2}$ denote the vacuum expectation values of the neutral components of $\Phi_{1}$ and $\Phi_{2}$, respectively, and $\alpha$ denotes the mixing angle of the two $C P$-even neutral Higgs bosons. In this parametrisation the tree-level couplings of the Higgs bosons to SM vector bosons and fermions only depend on $\tan \beta$ and $\beta-\alpha$. The couplings of the light $C P$-even Higgs boson $h$ are SM-like for $\beta-\alpha=\pi / 2$. We call this the alignment limit. To express the quartic couplings $\lambda_{i}$ in terms of the physical parameters (2) we use the tree-level relation

$$
v_{1}^{2}+v_{2}^{2}=v^{2}=\frac{M_{W}^{2}}{\pi \alpha_{\mathrm{em}}}\left(1-\frac{M_{W}^{2}}{M_{Z}^{2}}\right)
$$

with $M_{Z}=91.1878 \mathrm{GeV}, M_{W}=80.3693 \mathrm{GeV}$ and $\alpha_{\mathrm{em}} \equiv \alpha_{\mathrm{em}}\left(M_{Z}\right)=1 / 128.9529$ [53].

To compare the different triple Higgs couplings in the 2HDM with the SM triple Higgs coupling we define the ratios

$$
c_{\phi_{1} \phi_{2} \phi_{3}}=\frac{g_{\phi_{1} \phi_{2} \phi_{3}}^{2 \mathrm{HDM}}}{g_{h h h}^{\mathrm{SM}}},
$$

where $\phi_{1}, \phi_{2}, \phi_{3} \in\left\{h, H, A, H^{ \pm}\right\}, g_{\phi_{1} \phi_{2} \phi_{3}}^{2 \mathrm{HDM}}$ denotes the corresponding $2 \mathrm{HDM}$ triple Higgs coupling and $g_{h h h}^{\mathrm{SM}}$ denotes the SM triple Higgs coupling with a fixed SM Higgs mass of $126 \mathrm{GeV}$. In terms of the parameters in (2) these ratios are

$$
\begin{aligned}
c_{h h h}= & \frac{\cos ^{3} \alpha}{\sin \beta}-\frac{\sin ^{3} \alpha}{\cos \beta}-\frac{m_{12}^{2}}{m_{h}^{2}} \cos (\beta-\alpha)\left(\frac{\cos ^{2} \alpha}{\sin ^{2} \beta}-\frac{\sin ^{2} \alpha}{\cos ^{2} \beta}\right), \\
c_{H H H}= & \frac{m_{H}^{2}}{m_{h}^{2}}\left(\frac{\sin ^{3} \alpha}{\sin \beta}+\frac{\cos ^{3} \alpha}{\cos \beta}\right)-\frac{m_{12}^{2}}{m_{h}^{2}} \sin (\beta-\alpha)\left(\frac{\cos ^{2} \alpha}{\cos ^{2} \beta}-\frac{\sin ^{2} \alpha}{\sin ^{2} \beta}\right), \\
c_{h h H}= & \frac{\cos (\beta-\alpha)}{\cos \beta \sin \beta}\left[\frac{\cos \alpha \sin \alpha}{3}\left(2+\frac{m_{H}^{2}}{m_{h}^{2}}\right)+\frac{m_{12}^{2}}{m_{h}^{2}}\left(\frac{1}{3}-\frac{\cos \alpha \sin \alpha}{\cos \beta \sin \beta}\right)\right], \\
c_{h H H}= & \frac{\sin (\beta-\alpha)}{\cos \beta \sin \beta}\left[-\frac{\cos \alpha \sin \alpha}{3}\left(1+2 \frac{m_{H}^{2}}{m_{h}^{2}}\right)+\frac{m_{12}^{2}}{m_{h}^{2}}\left(\frac{1}{3}+\frac{\cos \alpha \sin \alpha}{\cos \beta \sin \beta}\right)\right], \\
c_{h H^{+} H^{-}}= & \frac{1}{3}\left[-\sin (\beta-\alpha)\left(1-2 \frac{m_{H^{ \pm}}^{2}}{m_{h}^{2}}\right)+\left(\frac{\cos \alpha}{\sin \beta}-\frac{\sin \alpha}{\cos \beta}\right)\left(1-\frac{1}{\cos \beta \sin \beta} \frac{m_{12}^{2}}{m_{h}^{2}}\right)\right],
\end{aligned}
$$




$$
\begin{aligned}
c_{H H^{+} H^{-}} & =\frac{1}{3}\left[-\cos (\beta-\alpha)\left(\frac{m_{H}^{2}}{m_{h}^{2}}-2 \frac{m_{H^{ \pm}}^{2}}{m_{h}^{2}}\right)+\left(\frac{\cos \alpha}{\cos \beta}+\frac{\sin \alpha}{\sin \beta}\right)\left(\frac{m_{H}^{2}}{m_{h}^{2}}-\frac{1}{\cos \beta \sin \beta} \frac{m_{12}^{2}}{m_{h}^{2}}\right)\right], \\
c_{h A A} & =\frac{1}{3}\left[-\sin (\beta-\alpha)\left(1-2 \frac{m_{A}^{2}}{m_{h}^{2}}\right)+\left(\frac{\cos \alpha}{\sin \beta}-\frac{\sin \alpha}{\cos \beta}\right)\left(1-\frac{1}{\cos \beta \sin \beta} \frac{m_{12}^{2}}{m_{h}^{2}}\right)\right], \\
c_{H A A} & =\frac{1}{3}\left[-\cos (\beta-\alpha)\left(\frac{m_{H}^{2}}{m_{h}^{2}}-2 \frac{m_{A}^{2}}{m_{h}^{2}}\right)+\left(\frac{\cos \alpha}{\cos \beta}+\frac{\sin \alpha}{\sin \beta}\right)\left(\frac{m_{H}^{2}}{m_{h}^{2}}-\frac{1}{\cos \beta \sin \beta} \frac{m_{12}^{2}}{m_{h}^{2}}\right)\right] .
\end{aligned}
$$

These formluae agree with the triple Higgs couplings from [54]. In the alignment limit $\beta-\alpha=\frac{\pi}{2}$ these expressions simplify to

$$
\begin{aligned}
c_{h h h}= & 1, \\
c_{h h H}= & 0, \\
c_{h X X}= & \frac{1}{3}\left(1+2 \frac{m_{X}^{2}}{m_{h}^{2}}-\frac{2}{\cos \beta \sin \beta} \frac{m_{12}^{2}}{m_{h}^{2}}\right) \\
c_{H X X}= & \frac{1}{3}(\tan \beta-\cot \beta) \\
& \cdot\left(\frac{m_{H}^{2}}{m_{h}^{2}}-\frac{1}{\cos \beta \sin \beta} \frac{m_{12}^{2}}{m_{h}^{2}}\right) \\
& \cdot \begin{cases}3 \quad \text { for } X=H, H^{ \pm} \\
1 \quad \text { for } X=A, H^{ \pm} .\end{cases}
\end{aligned}
$$

\section{THEORETICAL AND EXPERIMENTAL CONSTRAINTS}

For a detailed discussion of the theoretical and experimental constraints included in our analysis we refer to our previous paper [31]. Here we only briefly list the included constraints and comment on some updates and improvements which we made since the previous paper [31. The theoretical constraints are

- positivity of the Higgs potential [55,

- stability of the vacuum [56] and

- perturbativity of the Higgs self-couplings.

The perturbativity constraint is implemented in our analysis by requiring

$$
\|16 \pi S\|<\Lambda_{\max },
$$

where $S$ is the tree-level scattering matrix for Higgs and longitudinal gauge bosons, as defined in [57], and the matrix norm $\|\cdot\|$ is the magnitude of the largest eigenvalue. The unitarity of the tree-level $S$-matrix, implemented in a partial-wave analysis of the two-particle Fock states involving Higgs or longitudinal gauge bosons, requires $\left\|S_{\phi \phi}\right\| \leq 1$, i.e. $\Lambda_{\max }=16 \pi[58$ ]. This bound has been studied for the 2HDM in [57, 59, 62] and allows the $\lambda_{i}$ in (1) to be as large as a few multiples of $\pi$. For the SM, the unitarity analysis has been taken to the two-loop level in 63. In 64] upper bounds on the SM Higgs coupling have been derived by imposing certain consistency conditions on the perturbative series (order-by-order reduction of scheme and scale dependences). Both analyses have resulted in significantly tighter upper bounds on the on Higgs quartic coupling which, for the SM, corresponds roughly to $\Lambda_{\max }=2 \pi$. In this paper we present results for two choices of $\Lambda_{\max }$ : a loose bound $\Lambda_{\max }=16 \pi$ and a tight bound $\Lambda_{\max }=2 \pi$. We advocate the use of the tight bound. The loose bound is only included to show the sensitivity of certain features in our plots to the implementation of the perturbativity constraint and to facilitate comparisons with studies that only use tree-level unitarity to constrain the size of the quartic Higgs couplings.

The experimental constraints included in our analysis are

- the signal strengths of the light $C P$ even Higgs boson $h$, as measured by the ATLAS [3] and CMS 65] collaborations,

- the CMS exclusion limits for a heavy Higgs decaying into $W W, Z Z$ [66] or $\tau \tau$ 67. final states,

- the full set of electroweak precision observables 68] (see [31] for details) and

- the flavour constraints relevant for the low $\tan \beta$ region, i.e. the constraints from the 
mass splitting $\Delta m_{B_{s}}$ of the neutral $B_{s}$ system and the branching fraction $\operatorname{Br}(B \rightarrow$ $\left.X_{s} \gamma\right)$.

Compared to our previous analysis [31], the signal strength inputs have been improved by including the correlations between the different Higgs production mechanisms. To this end, we compute for each final state $X \in$ $\{\gamma \gamma, Z Z, W W, b \bar{b}, \tau \tau\}$ the signal strengths

$$
\begin{aligned}
\mu_{\mathrm{ggF}+\mathrm{ttH}}^{X} & =\frac{\left(\sigma_{\mathrm{ggF}}^{2 \mathrm{HDM}}+\sigma_{\mathrm{ttH}}^{2 \mathrm{HDM}}\right) \operatorname{Br}(h \rightarrow X)^{2 \mathrm{HDM}}}{\left(\sigma_{\mathrm{ggF}}^{\mathrm{SM}}+\sigma_{\mathrm{ttH}}^{\mathrm{SM}}\right) \operatorname{Br}(h \rightarrow X)^{\mathrm{SM}}}, \\
\mu_{\mathrm{VBF}+\mathrm{VH}}^{X} & =\frac{\left(\sigma_{\mathrm{VBF}}^{2 \mathrm{HDM}}+\sigma_{\mathrm{VH}}^{2 \mathrm{HDM}}\right) \operatorname{Br}(h \rightarrow X)^{2 \mathrm{HDM}}}{\left(\sigma_{\mathrm{VBF}}^{\mathrm{SM}}+\sigma_{\mathrm{VH}}^{\mathrm{SM}}\right) \operatorname{Br}(h \rightarrow X)^{\mathrm{SM}}},
\end{aligned}
$$

where the subscripts ' $g g F$ ', 'ttH', 'VBF' and 'VH' stand for 'gluon fusion', ' $t \bar{t}$ associated production', 'vector boson fusion' and 'Higgsstrahlung', respectively and the superscripts indicate the model in which the corresponding quantity is evaluated. The central values, errors and correlations of the $\mu_{\mathrm{ggF}+\mathrm{ttH}}^{X}$ and $\mu_{\mathrm{VBF}+\mathrm{VH}}^{X}$ are then extracted from Fig. 2 of [3] and Fig. 4 of 65, respectively. To compute the Higgs production cross sections and branching fractions in the $2 \mathrm{HDM}$ we follow the recommendations of [69]. In particular, we now compute the gluon fusion production cross sections for 2HDM Higgs bosons by scaling the top-loop and bottom-loop contributions and their interference with appropriate $2 \mathrm{HDM} / \mathrm{SM}$ ratios of Higgs-top and Higgsbottom couplings. The constraints from heavy Higgs searches in the $W W$ and $Z Z$ final states were improved by using the combined limits from Fig. 10 of 66]. In addition, we now use the model-independent limits from heavy Higgs searches in the $\tau \tau$ final state from [67] (median expected limits from Tabs. 7 and 8). Finally, our implementation of the electroweak precision observables now uses the updated result [70] for the SM prediction of $R_{b}$.

Our analysis makes use of several public codes. For the computation of effective $g g h$ and $h \gamma \gamma$ couplings and 2HDM contributions to electroweak precision observables we use FeynArts 3.5, FormCalc 7.0 and LoopTools 2.7 [71-73]. The SM contributions to the electroweak precision observables are computed with Zfitter
$6.43[74,76]$ and combined with the $2 \mathrm{HDM}$ contributions following the prescription in [77. SM Higgs production cross sections and partial widths were calculated with HIGLU $4.00[78$ and HDECAY 6.10 [79 81]. The latter code was also used to calculate 2HDM branching fractions. The fits were done with the myFitter framework 82 which in turn uses Dvegas 2.0.3 [83 85] for adaptive parameter scans. The fit results were cross-checked with an independent implementation in the CKMfitter framework [86].

\section{FIT RESULTS}

\section{A. Couplings to Vector Bosons and Fermions}

The tree-level couplings of the 2HDM Higgs bosons to vector bosons and fermions are completely determined by the parameters $\tan \beta$ and $\beta-\alpha$. Fig. 2 shows the regions in the $\tan \beta$ $(\beta-\alpha)$-plane which are allowed at $1 \sigma, 2 \sigma$ and $3 \sigma$ for tight (blue) and loose (green) perturbativity bound. For each combination of $\tan \beta$ and $\beta-\alpha$ we perform a likelihood ratio test (LRT), i.e. we compute the difference $\Delta \chi^{2}$ of minimum $\chi^{2}$-values obtained in the fit with all parameters free and the fit with $\tan \beta$ and $\beta-\alpha$ fixed. The number of standard deviations (sigmas) in this paper are two-sided $Z$-scores and are calculated in the asymptotic limit, i.e. by assuming that $\Delta \chi^{2}$ has a chi-square distribution with two degrees of freedom. Under this assumption the $\Delta \chi^{2}$ values corresponding to $1 \sigma, 2 \sigma$ and $3 \sigma$ are $2.3,6.2$ and 11.8, respectively.

We see that at $1 \sigma$ the value of $\beta-\alpha$ must be fairly close to $\pi / 2$ - the alignment limit where the $h$ couplings are SM-like. At the $1 \sigma$ level deviations of more than $0.01 \pi$ are only possible for $\tan \beta$ between 0.8 and 8 . The loose perturbativity bound admits slightly larger deviations of $\beta-\alpha$ from $\pi / 2$ for $\tan \beta<2$. For $\tan \beta>2$ and $\beta-\alpha \in[0.45 \pi, 0.55 \pi]$ there is essentially no difference between the two perturbativity bounds. There is an additional "island" in the $\tan \beta$ $(\beta-\alpha)$-plane where $\beta-\alpha$ can be as small as $0.3 \pi$. For the loose perturbativity bound this island is allowed at $2 \sigma$. For the tight bound it 


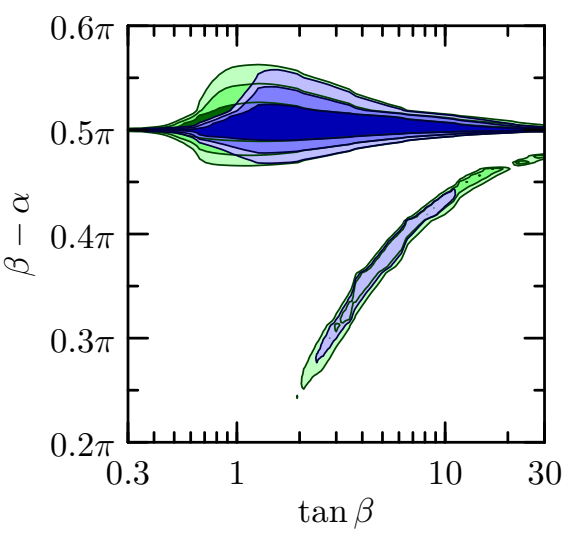

FIG. 2. Allowed region in the $\tan \beta$ - $(\beta-\alpha)$-plane. Shown are in blue the regions allowed at $1 \sigma$ (dark), $2 \sigma$ (medium) and $3 \sigma$ (light) for the tight perturbativity bound $\left(\Lambda_{\max }=2 \pi\right)$ as well as the corresponding regions for the loose perturbativity bound $\left(\Lambda_{\max }=16 \pi\right)$ in green. Note that the lower strip is allowed at $2 \sigma$ for $\Lambda_{\max }=16 \pi$ but only at $3 \sigma$ for $\Lambda_{\max }=2 \pi$.

is only allowed at $3 \sigma$. As discussed in [31], the best-fit points in this island have an enhanced effective $g g h$ coupling and a reduced effective $h \gamma \gamma$ coupling. They are allowed at $1 \sigma$ by the Higgs signal strengths but disfavoured by the flavour and electroweak precision constraints, since they exhibit rather small values of $m_{H^{ \pm}}$.

\section{B. Triple Higgs Couplings}

¿From 6) we know that $c_{h h h}=1$ in the alignment limit. Thus, deviations of the triple- $h$ coupling from its $\mathrm{SM}$ value, i.e. $c_{h h h} \neq 1$, imply $\beta-\alpha \neq \pi / 2$ and therefore only appear in conjunction with corresponding deviations of $h$ couplings to fermions and gauge bosons. The observed Higgs signal strengths can therefore be used to constrain the possible deviations of the triple $h$ coupling in the 2HDM. To calculate these constraints, we perform a LRT of the $2 \mathrm{HDM}$ with $c_{h h h}$ fixed. Fig. $3 \mathrm{a}$ shows the $p$ values of the test as a function of the value of $c_{h h h}$. The $p$-values were computed by assuming a chi-square distribution with one degree of freedom for $\Delta \chi^{2}$. For the loose perturbativity bound (dashed line) we see that the magnitude of $c_{h h h}$ can reach 1.7 at $2 \sigma$. A reduction of the coupling to zero is also possible at $2 \sigma$. At $1 \sigma$ (and for $\Lambda_{\max }=16 \pi$ ) the magnitude of $c_{h h h}$ cannot exceed 1 and must be bigger than 0.25 . The best-fit scenarios outside the $1 \sigma$ region for the loose perturbativity bound feature quartic couplings $\lambda_{i}$ (c.f. (1)) with magnitude above 10. If we impose the tight perturbativity bound the magnitude of $c_{h h h}$ can no longer exceed 1 at $3 \sigma$. It can be reduced to 0.72 at $1 \sigma$, to 0.56 at $2 \sigma$ and to 0.4 at $3 \sigma$.

The coupling ratio $c_{h h H}$ vanishes in the alignment limit and is thus also strongly constrained by the Higgs signal strengths. Fig. 3b shows the ranges for $c_{h h H}$ allowed at the 1,2 and $3 \sigma$ levels as a function of $m_{H}$. For $m_{H} \gtrsim 400 \mathrm{GeV}$ the $1 \sigma$ contour for the loose perturbativity bound coincides with the corresponding contour for the tight perturbativity bound. The best-fit points inside this contour are close to the alignment limit and mainly constrained by the Higgs signal strengths. For the tight perturbativity bound the allowed region does not increase significantly when we go to the 2 or $3 \sigma$ level, but it does for the loose perturbativity bound. This is due to the fact that the loose perturbativity bound allows scenarios far away from the alignment limit at $2 \sigma$, as seen in Fig. 2 .

The remaining triple Higgs couplings are not fixed in the alignment limit and their allowed range is mainly determined by the perturbativity requirement. Consequently, the allowed ranges for the tight and loose perturbativity bounds differ substantially. Figs. 3 - $f$ show the ranges allowed at 1, 2 and $3 \sigma$ as a function of the heavy Higgs mass. While the loose perturbativity bound allows enhancement factors of 20 or more in the displayed mass range, the enhancement factors for the tight perturbativity bound cannot exceed 6 in magnitude. The symmetry of the plots is due to the following property of the triple Higgs couplings: if we shift $\beta-\alpha$ by $\pi$ and keep all other parameters in (2) fixed all triple Higgs couplings flip their sign but keep the same magnitude. Such a shift in $\beta-\alpha$ corresponds to a field re-definition $(h, H) \rightarrow(-h,-H)$ and therefore has no physical consequence. 

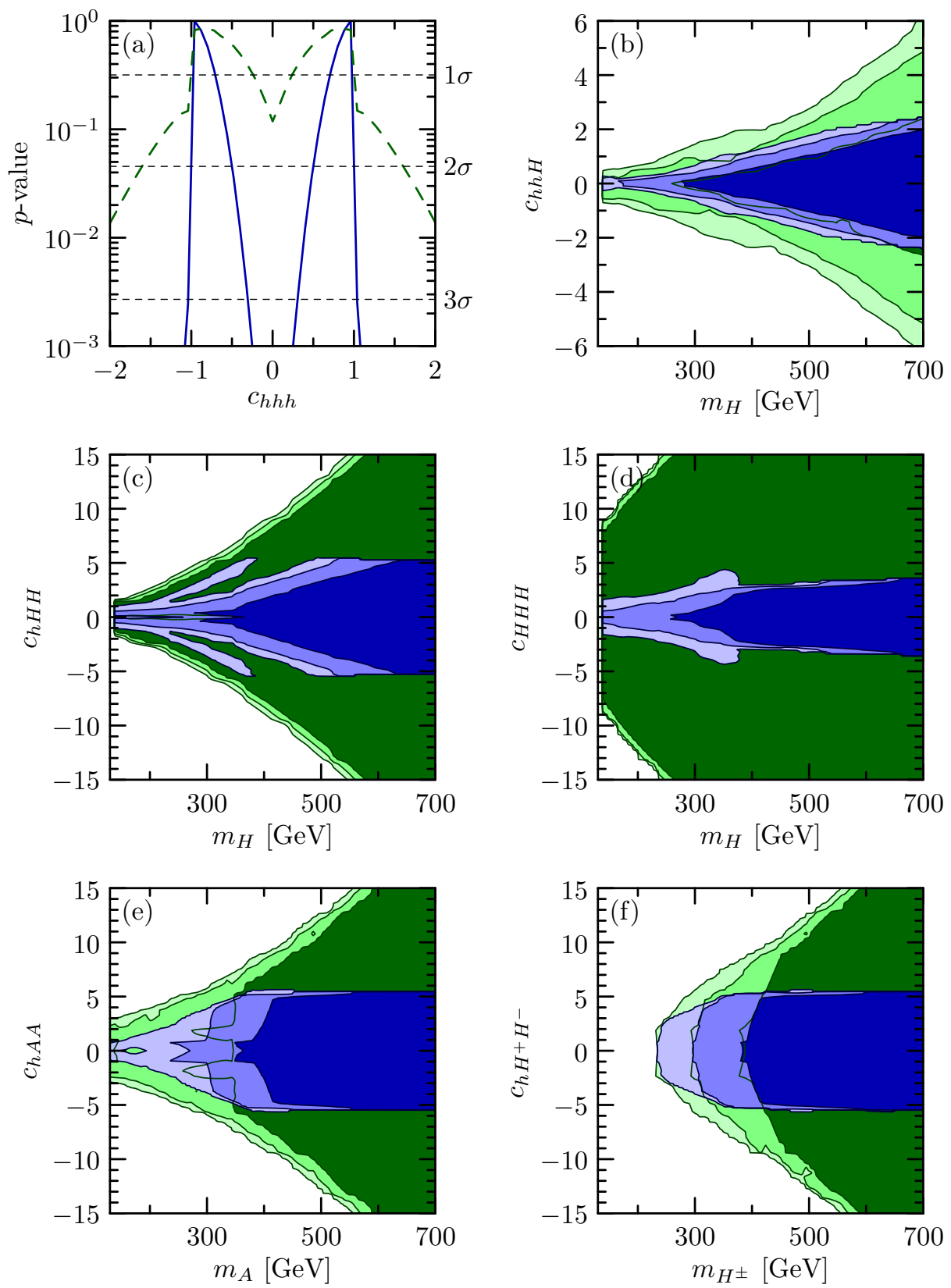

FIG. 3. (a) $p$-value of the $2 \mathrm{HDM}$ with a fixed $2 \mathrm{HDM} / \mathrm{SM}$ ratio $c_{h h h}$ of triple $h$ couplings (see (5) for the complete expression) as a function of $c_{h h h}$. The solid line corresponds to the tight perturbativity bound $\left(\Lambda_{\max }=2 \pi\right)$ and the dashed line to the loose bound $\left(\Lambda_{\max }=16 \pi\right)$. (b)-(f) Allowed ranges for the $2 \mathrm{HDM} / \mathrm{SM}$ triple Higgs coupling ratios as a function of the corresponding heavy Higgs mass. Shown are in blue the regions allowed at $1 \sigma$ (dark), $2 \sigma$ (medium) and $3 \sigma$ (light) for the tight perturbativity bound $\left(\Lambda_{\max }=2 \pi\right)$ as well as the corresponding regions for the loose perturbativity bound $\left(\Lambda_{\max }=16 \pi\right)$ in green. The $p$-values in (a) were computed by assuming a chi-square distribution for the test statistic with one degree of freedom. For the significances in (b)-(f) we assumed a chi-square distribution with two degrees of freedom.

\section{Branching Fractions}

The search for additional Higgs resonances in the 2HDM is complicated by the possible existence of tree-level decays into lighter Higgs bosons like $H \rightarrow h h$ or $A \rightarrow Z h$. If kinematically allowed, these decays compete with the standard decay modes $H, A \rightarrow X_{\text {std }}$, where $X_{\text {std }}$ stands for 
all 'standard' final states which do not contain another Higgs boson (i.e. $g g, \gamma \gamma, W W, Z Z, Z \gamma$, $t \bar{t}, b \bar{b}, \tau \tau$ etc.). Of course the non-standard decays may also be regarded as alternative discovery modes for the heavy Higgs bosons or as additional sources of background for the observation of the light SM-like Higgs. To estimate the possible size of the non-standard branching fractions of the heavy Higgs bosons we show the 1,2 and $3 \sigma$ allowed regions in the $m_{H}-\operatorname{Br}\left(H \rightarrow X_{\text {std }}\right)$ plane in Fig. 4 a. Only the results for the tight perturbativity bound are displayed.

To achieve the largest non-standard branching fractions the mass of the decaying Higgs boson has to be below the $t \bar{t}$ threshold, so that the non-standard decays do not have to compete with the large $t \bar{t}$ partial width. In this mass region the branching fraction $\operatorname{Br}\left(H \rightarrow X_{\text {std }}\right)$ can drop below $40 \%$ at the $2 \sigma$ level. This is due to the non-standard $H \rightarrow h h$ decay. The branching fraction $\operatorname{Br}\left(A \rightarrow X_{\text {std }}\right)$ can drop below $1 \%$ at the $2 \sigma$ level if $m_{A}$ is in the narrow window between $320 \mathrm{GeV}$ and $2 m_{t}$. This is due to a non-trivial interplay of all constraints in our fit: since couplings involving the light Higgs cannot be enhanced very much, the largest nonstandard partial widths are obtained for heavyto-heavy decay modes like $A \rightarrow Z H$. For this decay to be open we need a mass splitting of more than $90 \mathrm{GeV}$ between $A$ and $H$. To close the competing $A \rightarrow t \bar{t}$ decay channel, $m_{A}$ must be below $2 m_{t} \approx 350 \mathrm{GeV}$, which puts $m_{H}$ below $260 \mathrm{GeV}$. But electroweak precision constraints require one of the two heavy neutral Higgs bosons to be almost degenerate with the charged Higgs, and flavour constraints force the charged Higgs mass to be above approximately $320 \mathrm{GeV}$. Since the charged Higgs cannot be degenerate with $H$ (as $m_{H}$ is below $260 \mathrm{GeV}$ ) it must be degenerate with $A$, and this means $m_{A}$ must be between $320 \mathrm{GeV}$ and $2 m_{t}$ to achieve the largest $A \rightarrow Z H$ partial width. For $m_{A}$ above $2 m_{t}$ the branching fraction $\operatorname{Br}\left(A \rightarrow X_{\text {std }}\right)$ can still be below $8 \%$ at the $2 \sigma$ level, but the lower limit increases with $m_{A}$. For $m_{A} \gtrsim 400 \mathrm{GeV}$ the $A \rightarrow H^{ \pm} W^{\mp}$ decays are possible without violating the constraint on $m_{H^{ \pm}}$from $\operatorname{Br}\left(\bar{B} \rightarrow X_{s} \gamma\right)$. As a result, standard branching fractions as low as $30 \%$ are allowed at
$1 \sigma$ for $m_{A} \approx 400 \mathrm{GeV}$

\section{PHENOMENOLOGY}

\section{A. Benchmarks}

The previous section has shown that today's measurements place severe constraints on parameters of the $2 \mathrm{HDM}$ of type II to be probed in Run II of the LHC and at a future linear collider. Our results can serve as input for studies of collider signals in the type-II 2 HDM. In practice, these are only feasible for a small number of benchmark points. The benchmarks should maximise the collider signal under investigation while being in agreement with all existing constraints. Therefore, the criteria by which the benchmark points should be selected depend on the physics one wants to investigate. In searches for (non-resonant) Higgs pair production one is generally interested in scenarios with large triple Higgs couplings, while searches for heavy Higgs resonances with non-standard decay modes need benchmarks where the branching fractions of the non-standard decays are as large as possible. In this section we provide benchmark points for the type-II $2 \mathrm{HDM}$ which are tailored for these two types of new physics searches.

Our benchmarks for large triple-Higgs couplings are summarised in Tab. I. The benchmarks were constructed from best-fit parameters corresponding to points inside the $2 \sigma$ allowed regions in Figs. $3 \mathrm{z}$-e and as close as possible to their edges. As such, they agree with all constraints in our fit at the $2 \sigma$ level. Benchmark a-1 corresponds to Fig. $3 a$ and is chosen to minimise the absolute value of $c_{h h h}$. Benchmarks b- 1 to b- 4 correspond to Fig. $3 \mathrm{~b}$ and maximise $\left|c_{h h H}\right|$ for different fixed values of $m_{H}$. The remaining benchmarks in Tab. I were constructed from Figs. 3 r-e in an analogous way.

Tab. III contains benchmarks featuring large non-standard decay rates of the heavy Higgs bosons $H$ and $A$. They were constructed from best-fit points inside the $2 \sigma$ contours in Fig. 4 and as close as possible to their edges. The branching fractions of $H$ and $A$ for these benchmark points are given in Tabs. III and IV, re- 

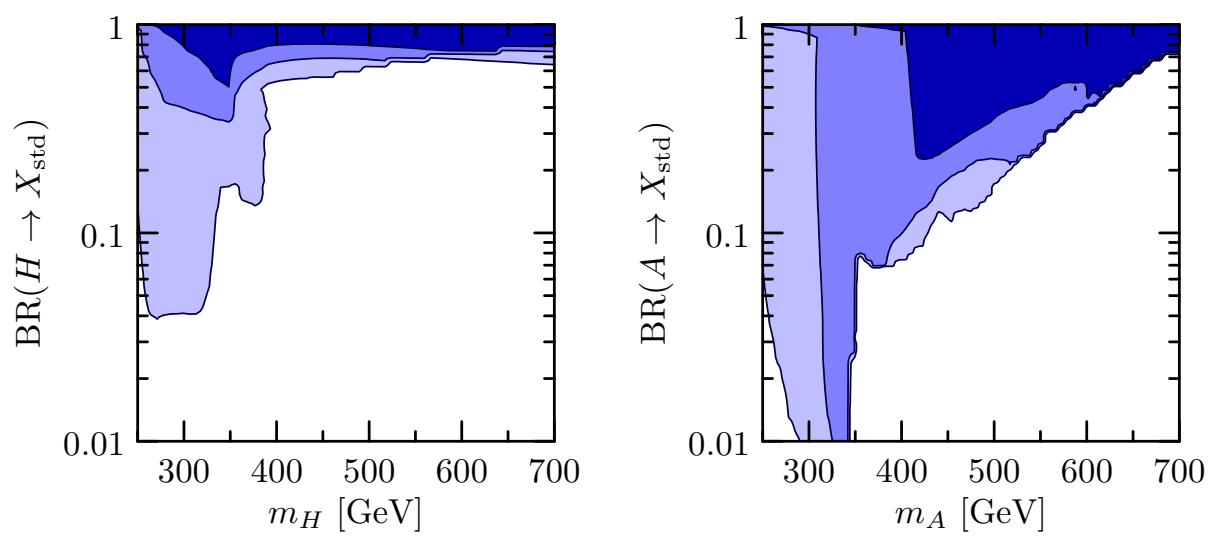

FIG. 4. Allowed ranges for the branching fractions of heavy neutral Higgs bosons into 'standard' final states (i.e. states which do not contain another Higgs boson) as a function of the corresponding heavy Higgs mass. Shown are the regions allowed at $1 \sigma$ (dark), $2 \sigma$ (medium) and $3 \sigma$ (light) for the tight perturbativity bound $\left(\Lambda_{\max }=2 \pi\right)$.

\begin{tabular}{|c|c|c|c|c|c|c|}
\hline \multicolumn{3}{|c|}{$\tan \beta(\beta-\alpha) / \pi m_{l}$} & \multirow{2}{*}{$\begin{array}{r}\mathrm{GeV}] \\
700\end{array}$} & \multirow{2}{*}{$\frac{m_{A}[\mathrm{GeV}]}{700}$} & \multirow{2}{*}{$m_{H^{ \pm}}$} & {$[\mathrm{GeV}] m_{12}^{2}\left[\mathrm{GeV}^{2}\right]$} \\
\hline$a-1$ & 1.50 & 0.529 & & & & 180000 \\
\hline b-1 & 2.52 & 0.511 & 200 & 383 & 383 & 14300 \\
\hline b-2 & 2.23 & 0.525 & 300 & 444 & 445 & 32700 \\
\hline b-3 & 1.73 & 0.533 & 400 & 502 & 503 & 62900 \\
\hline b-4 & 1.74 & 0.533 & 600 & 619 & 579 & 126000 \\
\hline$c-1$ & 2.22 & 0.505 & 200 & 337 & 337 & 12000 \\
\hline$c-2$ & 1.88 & 0.509 & 300 & 361 & 365 & 27800 \\
\hline$c-3$ & 1.49 & 0.518 & 400 & 350 & 407 & 47100 \\
\hline$c-4$ & 1.25 & 0.522 & 600 & 491 & 600 & 123000 \\
\hline$d-1$ & 2.78 & 0.503 & 200 & 319 & 320 & 10400 \\
\hline$d-2$ & 2.17 & 0.507 & 300 & 350 & 347 & 26500 \\
\hline$d-3$ & 1.85 & 0.503 & 400 & 350 & 404 & 53100 \\
\hline$d-4$ & 2.40 & 0.520 & 600 & 634 & 587 & 114000 \\
\hline $\mathrm{e}-2$ & 5.34 & 0.502 & 250 & 300 & 307 & 10700 \\
\hline e-3 & 4.90 & 0.502 & 229 & 400 & 399 & 10300 \\
\hline e-4 & 6.45 & 0.502 & 498 & 600 & 601 & 37530 \\
\hline
\end{tabular}

TABLE I. Benchmark scenarios with enhanced/reduced triple Higgs couplings. Benchmark a-1 approximates the best-fit scenario with reduced $h h h$ coupling at the edge of the $2 \sigma$ interval in Fig. 3a. Benchmarks b-1 to b-4 approximate the best-fit scenarios associated with points on the $2 \sigma$ contour in Fig. 3 3 . Benchmarks c-1 to e- 4 are related to Figs. 3 ; , $3 \mathrm{~d}$ and $3 \mathrm{~s}$ in an analogous way. All points are allowed at the $2 \sigma$ level.

spectively. We see that for scenarios that minimize $\operatorname{Br}\left(H \rightarrow X_{\text {std }}\right)$ (at the $2 \sigma$ level) the largest non-standard decay rate is $\operatorname{Br}(H \rightarrow h h)$ while for scenarios that minimize $\operatorname{Br}\left(A \rightarrow X_{\text {std }}\right)$ the largest non-standard decay rate is $\operatorname{Br}(A \rightarrow Z H)$.

\section{B. Higgs Pair Production in the Gluon Fusion Channel}

As we have seen in Section 4 the triple Higgs coupling between the light Higgs state cannot be enhanced. However, this does not mean that the gluon fusion cross section $\sigma(g g \rightarrow h h)$ cannot be enhanced with respect to the SM. In the sec- 


\begin{tabular}{|c|c|c|c|c|c|c|}
\hline \multicolumn{3}{|c|}{$\tan \beta(\beta-\alpha) / \pi$} & $\mathrm{GeV}]$ & $m_{A}[\mathrm{GeV}]$ & $m_{H^{ \pm}}[\mathrm{GeV}]$ & $m_{12}^{2}\left[\mathrm{GeV}^{2}\right]$ \\
\hline H-1 & 1.75 & 0.522 & 300 & 441 & 442 & $2 \quad 38300$ \\
\hline $\mathrm{H}-2$ & 2.00 & 0.525 & 340 & 470 & 471 & 44400 \\
\hline $\mathrm{H}-3$ & 4.26 & 0.519 & 450 & 546 & 548 & 43200 \\
\hline $\mathrm{H}-4$ & 4.28 & 0.513 & 600 & 658 & 591 & 76900 \\
\hline A-1 & 4.61 & 0.505 & 346 & 300 & 345 & 23600 \\
\hline A-2 & 2.74 & 0.503 & 131 & 340 & 339 & 6200 \\
\hline A-3 & 7.02 & 0.508 & 290 & 450 & 446 & 11700 \\
\hline A-4 & 7.44 & 0.504 & 490 & 600 & 598 & 31620 \\
\hline
\end{tabular}

TABLE II. Benchmark scenarios with large non-standard decay rates of heavy Higgs bosons. Benchmarks H-1 to H-4 approximate the best-fit scenarios associated with points on the lower $2 \sigma$ contour in Fig. 4 a. Benchmarks A-1 to A-4 are related to Fig. $4 \mathrm{~b}$ in an analogous way. All points have a $\Delta \chi^{2}$ less than 6.2 , i.e. are allowed at $2 \sigma$ in a LRT with two degrees of freedom.

\begin{tabular}{lcrrrrrrr}
\hline \hline & $m_{H}[\mathrm{GeV}]$ & $t \bar{t}$ & $b \bar{b}$ & $\tau \tau$ & $W W$ & $Z Z$ & $g g$ & $h h$ \\
\hline $\mathrm{H}-1$ & 300 & 0.1 & 10.6 & 1.2 & 18.9 & 8.4 & 1.8 & 59.0 \\
$\mathrm{H}-2$ & 340 & 1.4 & 7.0 & 0.8 & 17.8 & 8.1 & 1.2 & 63.7 \\
$\mathrm{H}-3$ & 450 & 51.6 & 9.2 & 1.1 & 6.0 & 2.8 & 0.2 & 29.0 \\
$\mathrm{H}-4$ & 600 & 64.0 & 7.3 & 0.9 & 4.4 & 2.1 & 0.2 & 21.1 \\
\hline $\mathrm{A}-1$ & 346 & 1.5 & 81.0 & 9.5 & 1.5 & 0.7 & 0.6 & 5.1 \\
$\mathrm{~A}-2$ & 131 & & 90.4 & 8.8 & & & 0.6 & \\
$\mathrm{~A}-3$ & 290 & & 85.2 & 9.7 & 1.0 & 0.5 & 0.2 & 3.4 \\
$\mathrm{~A}-4$ & 490 & 32.5 & 56.3 & 7.0 & 0.6 & 0.3 & 0.1 & 3.0 \\
\hline \hline
\end{tabular}

TABLE III. Branching fractions (in percent) of the heavy scalar Higgs boson $H$ for the benchmarks from Tab. II

\begin{tabular}{lcrrrrrrr}
\hline \hline \multicolumn{3}{c}{$m_{A}[\mathrm{GeV}]$} & $t \bar{t}$ & $b \bar{b}$ & $\tau \tau$ & $g g$ & $Z h$ & $Z H$ \\
\hline $\mathrm{H}-1$ & 441 & 74.9 & 0.3 & & 0.3 & 1.1 & 23.4 \\
$\mathrm{H}-2$ & 470 & 77.4 & 0.5 & 0.1 & 0.3 & 2.2 & 19.6 \\
$\mathrm{H}-3$ & 546 & 78.4 & 9.1 & 1.2 & 0.3 & 8.2 & 3.0 \\
$\mathrm{H}-4$ & 658 & 82.8 & 9.2 & 1.2 & 0.2 & 6.3 & \\
\hline $\mathrm{A}-1$ & 300 & 0.1 & 88.7 & 10.1 & 0.4 & 0.5 & \\
$\mathrm{~A}-2$ & 340 & 0.4 & 0.8 & 0.1 & 0.1 & & 98.6 \\
$\mathrm{~A}-3$ & 450 & 9.6 & 9.2 & 1.1 & & 0.3 & 79.7 \\
$\mathrm{~A}-4$ & 600 & 32.5 & 33.8 & 4.4 & 0.1 & 0.6 & 28.7 \\
\hline \hline
\end{tabular}

TABLE IV. Branching fractions (in percent) of the pseudoscalar Higgs boson $A$ for the benchmarks from Tab. I

ond diagram of Fig. 1, not only the light Higgs state $h$ contributes in the propagator, but also the heavy Higgs state $H$. For $m_{H}>2 m_{h}$ this diagram represents a resonant $H$ production with a subsequent non-standard decay into $h h$, while for $m_{H}<2 m_{h}$ it gives a non-resonant contribution to the $h h$ production cross section.

In the non-resonant case we expect large deviations of $\sigma(g g \rightarrow h h)$ from its SM value when $\left|c_{h h H}\right|$ is large and $m_{H}$ just below the $h h$ threshold. However, for $m_{H}<2 m_{h}$ the absolute value of $c_{h h H}$ is constrained to be below approximately 0.17 at the $2 \sigma$ level, as can be seen in Fig. 3b. To study such a scenario in detail we computed $\sigma(g g \rightarrow h h)$ for benchmark b-1 (see Tab. I). Using the set-up described in Ref. [39] and a modified version of HPAIR [42] with the type-II 2HDM implemented, we obtain $\sigma(g g \rightarrow h h)_{2 \mathrm{HDM}, \mathrm{b}-1}=30.3 \mathrm{fb}$ at the LHC with $14 \mathrm{TeV}$. This is a reduction of $10 \%$ compared to the SM value $\sigma(g g \rightarrow h h)_{\mathrm{SM}}=33.5 \mathrm{fb}$, and is due to the fact that the triangle diagram (c.f. Fig. 1) involving the heavy Higgs $H$ interferes destructively with the box diagram and the triangle diagram involving the light Higgs $h$. In addition, the $h h h$ and $h t \bar{t}$ couplings are slightly reduced in this scenario. These results indicate that a large enhancement is not possible in the non-resonant case.

In the resonant case we expect large enchancements of $\sigma(g g \rightarrow h h)$ when the $H \rightarrow h h$ decay has a large branching fraction. This situation is realised in benchmarks $\mathrm{H}-1$ to $\mathrm{H}-4$ (see Tab. II). Fig. 5 shows the $2 \mathrm{HDM} / \mathrm{SM}$ ratio of the $g g \rightarrow h h$ cross section as a function of the hadronic centre of mass energy for these benchmarks. The magnitude of $c_{h h h}$ is close to 1 for all these benchmarks, which means that the con- 


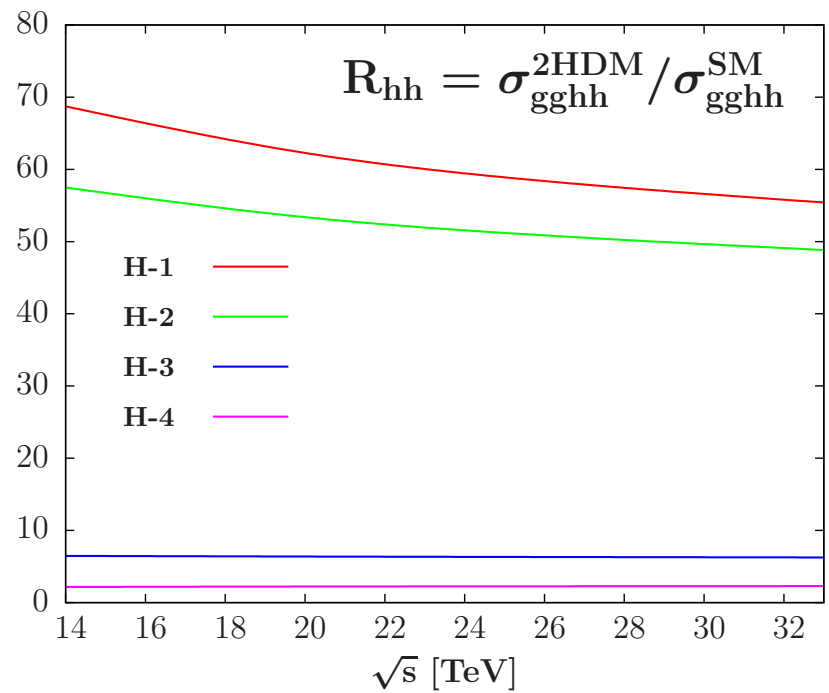

FIG. 5. Ratio between the gluon fusion cross section $\sigma(g g \rightarrow h h)$ at the LHC in 2HDM of type II using benchmark scenarios of type $\mathrm{H}$ in Tab. II] and the same cross section but within the SM, as a function of the center-of-mass energy $\sqrt{s}$ (in $\mathrm{TeV})$.

tribution from the diagram with the $s$-channel $h$ exchange is SM-like. We see that the $g g \rightarrow h h$ cross section can be enhanced by more than a factor 50 for benchmarks $\mathrm{H}-1$ and $\mathrm{H}-2$ and that benchmark H-3 still gives a factor 6 enhancement.

We also investigated the possibility that the only hints for heavy Higgs resonances at the $14 \mathrm{TeV}$ run of the LHC after $300 \mathrm{fb}^{-1}$ of data are in the $h h$ final state. Using an extrapolation of the current limits for heavy Higgs resonances in the $W W, Z Z$ and $\tau \tau$ channels based on the ratio of expected signal events we find that scenarios with heavy Higgs masses below the $t \bar{t}$ threshold can be ruled out (or discovered) by the standard searches. The ILC in its first stage at $\sqrt{s}=250 \mathrm{GeV}$ would impose even stronger bounds on additional Higgs resonances below the $t \bar{t}$ threshold, but provide little information about heavier resonances. Benchmarks $\mathrm{H}-3$ and $\mathrm{H}-4$ can easily evade the expected limits from both ILC and the LHC at $14 \mathrm{TeV}$ as they have all heavy Higgs bosons above the $t \bar{t}$ threshold. However, as seen in Fig. 5 they still lead to a noticable enhancement in the Higgs pair production rate.

\section{CONCLUSIONS}

The existing data from flavour, electroweak precision and Higgs physics imposes severe constraints on extended Higgs sectors and can be used to to put limits on the possible size of signals in future collider searches. In this paper we studied the bounds on triple Higgs couplings and branching fractions of non-standard decays of heavy Higgs bosons (i.e. decays into other Higgs bosons) in the context of the $C P$ conserving two-Higgs-doublet-model of type II. Our analysis uses current experimental constraints from flavour, electroweak precision and Higgs data as well as theoretical constraints such as positivity of the Higgs potential, vacuum stability and perturbativity. While our results were presented for two different implementations of the perturbativity bound, we advocate the use of the "tight" bound which requires the magnitude of the eigenvalues of the tree-level $\phi \phi \rightarrow \phi \phi$ $S$-matrix to be below $1 / 8$.

With regard to the questions (i) to (iv) raised in the introduction, our results may be summarised as follows:

i) assuming that the observed Higgs resonance is the light $C P$-even Higgs boson $h$ of the 2HDM, the data on Higgs signal strengths pushes the parameters of the 2HDM close to the alignment limit, where the couplings of $h$ are SM-like. In this limit, also the $h h h$ coupling is SM-like and the $h h H$ coupling vanishes. Deviations of these couplings from the alignment limit are therefore strongly constrained by the observed Higgs signal strengths. Using all available theoretical and experimental constraints we have shown that an enhancement of the hhh coupling is forbidden at the $3 \sigma$ level, while a reduction by a factor of 0.56 is possible at the $2 \sigma$ level (Fig. 3a).

ii) The allowed range for the $h h H$ coupling increases linearly with $m_{H}$ and then saturates at 2.5 times the size of the $\mathrm{SM} h h h$ coupling (Fig. 3b). The hHH, HHH, $h A A$ and $h H^{+} H^{-}$couplings are not fixed in the alignment limit and are therefore 
mostly constrained by the requirement of perturbativity. Their allowed ranges also increase and then saturate with the mass of the corresponding heavy Higgs boson. Using the tight perturbativity bound we find that these couplings can be at most 5.5 times as large as the SM $h h h$ coupling (Figs. 3r-f).

The results of our fits were condensed into a set of benchmark points (Tab. II) which agree with present experimental data at the $2 \sigma$ level and feature large triple Higgs couplings. These benchmarks can be used to study collider signatures for processes like Higgs pair production, where large triple Higgs couplings lead to enhanced signals.

iii) To estimate the possible suppression of heavy Higgs signals due to nonstandard decay modes (i.e. decays involving another Higgs boson) we have determined the allowed range of the standard branching fractions $\operatorname{Br}(H \rightarrow$ $\left.X_{\text {std }}\right)$ and $\operatorname{Br}\left(A \rightarrow X_{\text {std }}\right)$ (with $X_{\text {std }}=$ $g g, W W, Z Z, t \bar{t}, b \bar{b}, \tau \tau, \ldots)$ as a function of the decaying Higgs mass. We find that the strongest suppression of the standard decay modes occurs for heavy Higgs masses below the $t \bar{t}$ threshold. If $m_{H}$ is below $2 m_{t}$ the branching fraction $\operatorname{Br}\left(H \rightarrow X_{\text {std }}\right)$ can be reduced to $40 \%$ at the $2 \sigma$ level due to the competing $H \rightarrow h h$ decay. Above the $t \bar{t}$ threshold values of $\operatorname{Br}\left(H \rightarrow X_{\text {std }}\right)$ as low as $70 \%$ are still possible (Fig. (4). The branching fraction $\operatorname{Br}\left(A \rightarrow X_{\text {std }}\right)$ for the pseudo-scalar $A$ can be much smaller when competing heavy-to-heavy decays like $A \rightarrow Z H$ or $A \rightarrow W^{ \pm} H^{\mp}$ are kinematically allowed. This is due to the fact that the relevant triple Higgs couplings are not as strongly constrained as the couplings involving the light Higgs $h$. The strongest reductions of $\operatorname{Br}\left(A \rightarrow X_{\text {std }}\right)$ which are allowed at the $2 \sigma$ level occur when the $A \rightarrow Z H$ decay is open. for $m_{A}$ between $320 \mathrm{GeV}$ and $2 m_{t}$ this can lead to a reduction below $1 \%$.

To facilitate further studies of $2 \mathrm{HDM}$ sce- narios with large non-standard branching fractions we have provided benchmark points with maximal non-standard decay rates (Tab. II).

iv) To study the effect of the heavy Higgs boson $H$ in light Higgs pair production we have calculated the $g g \rightarrow h h$ cross section at the $14 \mathrm{TeV}$ LHC for our benchmark points b- 1 and $\mathrm{H}-1$ to H-4. For benchmark b-1 the heavy Higgs only gives a non-resonant contribution and actually leads to a reduction of the cross section compared to the SM. This is due to a destructive interference between the triangle diagram (c.f. Fig. 1) and the diagrams involving only the light Higgs $h$. For benchmarks $\mathrm{H}-1$ to $\mathrm{H}-4$ the heavy Higgs is produced on-shell and then decays into an $h h$ pair. In scenarios $\mathrm{H}-1$ and $\mathrm{H}-2$ the SM $g g \rightarrow h h$ cross section is enhanced by more than a factor 50. For H-3 we still find an enhancement by a factor 6 (Fig. 5).

Using a naive extrapolation of the current limits for heavy Higgs resonances we find that benchmarks $\mathrm{H}-1$ and $\mathrm{H}-2$ can be verified or ruled out by standard $A \rightarrow \tau \tau$ searches with $300 \mathrm{fb}^{-1}$ of data at a $14 \mathrm{TeV}$ LHC. However, for benchmarks H-3 and $\mathrm{H}-4$ the heavy Higgs bosons would still be invisible to these searches and the only hint for their existence might be the enhanced $g g \rightarrow h h$ cross section.

It is often stated that a measurement of the hhh coupling may reveal new physics. In this paper we have found that this statement does not apply for the discrimination of the SM and the $2 \mathrm{HDM}$ of type II. Current data on the $h$ couplings to vector bosons and fermions (together with flavour and electroweak precision data) already constrain the maximally possible deviation of the $2 \mathrm{HDM}$ hhh coupling from the SM limit to be of the order of the experimental accuracy expected from $300 \mathrm{fb}^{-1}$ of $14 \mathrm{TeV}$ LHC data. This means that substantial improvements in the SM theoretical predictions and in experimental tools are required to discriminate between the type-II 2HDM and the SM by means 
of the triple $h$ coupling. While the $h$ pair production cross section in gluon fusion may be significantly enhanced in the 2HDM (see (iv) above), this feature is mostly independent of the size of the $h h h$ coupling.

\section{ACKNOWLEDGMENTS}

We thank Ansgar Denner, Rui Santos and Cédric Weiland for fruitful discussions and the CKMfitter group for allowing us to use their statistical analysis framework. The research leading to these results has received funding from the European Research Council under the European Union's Seventh Framework Programme (FP/2007-2013) / ERC Grant Agreement n. 279972. We also acknowledge sup- port by DFG through grants NI1105/2-1 and LE1246/9-1. J.B. acknowledges support by DFG through the SFB/TR-9 Computational Particle Physics.

\section{Note added}

After the completion of our work a similar study [87] appeared which focusses on the prospects of direct observation of the heavy Higgs bosons at the $14 \mathrm{TeV}$ LHC run in the context of $2 \mathrm{HDM}$ of type I and II and also discusses the case that the observed Higgs resonance is the heavy $C P$-even Higgs $H$. Our results concerning the allowed range of the $h h h$ coupling in the $2 \mathrm{HDM}$ of type II have been confirmed by the results of this new paper.
[1] S. Chatrchyan et al. (CMS Collaboration), Phys.Lett. B716, 30 (2012), arXiv:1207.7235 [hep-ex].

[2] G. Aad et al. (ATLAS Collaboration), Phys.Lett. B716, 1 (2012), arXiv:1207.7214 [hep-ex].

[3] ATLAS conference note, ATLAS-CONF-2013034 .

[4] S. Chatrchyan et al. (CMS Collaboration), JHEP 1306, 081 (2013), arXiv:1303.4571 [hep$\mathrm{ex}]$.

[5] P. W. Higgs, Phys.Rev.Lett. 13, 508 (1964),

[6] F. Englert and R. Brout, Phys.Rev.Lett. 13, 321 (1964).

[7] G. Guralnik, C. Hagen, and T. Kibble, Phys.Rev.Lett. 13, 585 (1964).

[8] T. Lee, Phys.Rev. D8, 1226 (1973).

[9] P. Ferreira, R. Santos, M. Sher, and J. P. Silva, (2011), arXiv:1112.3277 [hep-ph].

[10] K. Blum and R. T. D'Agnolo, Phys.Lett. B714, 66 (2012), arXiv:1202.2364 [hep-ph],

[11] L. Basso, A. Lipniacka, F. Mahmoudi, S. Moretti, P. Osland, et al., JHEP 1211, 011 (2012), arXiv:1205.6569 [hep-ph].

[12] H. Cheon and S. K. Kang, (2012), arXiv:1207.1083 [hep-ph].

[13] D. Carmi, A. Falkowski, E. Kuflik, T. Volansky, and J. Zupan, (2012), arXiv:1207.1718 [hep-ph]

[14] P. Ferreira, H. E. Haber, R. Santos, and J. P. Silva, (2012), arXiv:1211.3131 [hep-ph].

[15] A. Drozd, B. Grzadkowski, J. F. Gunion, and Y. Jiang, (2012), arXiv:1211.3580 [hep-ph].
[16] J. Chang, K. Cheung, P.-Y. Tseng, and T.-C. Yuan, Phys.Rev. D87, 035008 (2013), arXiv:1211.3849 [hep-ph],

[17] C.-Y. Chen and S. Dawson, (2013), arXiv:1301.0309 [hep-ph].

[18] A. Celis, V. Ilisie, and A. Pich, JHEP 1307, 053 (2013), arXiv:1302.4022 [hep-ph],

[19] P. P. Giardino, K. Kannike, I. Masina, M. Raidal, and A. Strumia, (2013), arXiv:1303.3570 [hep-ph].

[20] B. Grinstein and P. Uttayarat, (2013), arXiv:1304.0028 [hep-ph].

[21] J. Shu and Y. Zhang, (2013), arXiv:1304.0773 [hep-ph].

[22] A. Barroso, P. Ferreira, R. Santos, M. Sher, and J. P. Silva, (2013), arXiv:1304.5225 [hep-ph].

[23] G. Belanger, B. Dumont, U. Ellwanger, J. Gunion, and S. Kraml, Phys.Rev. D88, 075008 (2013), arXiv:1306.2941 [hep-ph]

[24] V. Barger, L. L. Everett, H. E. Logan, and G. Shaughnessy, Phys.Rev. D88, 115003 (2013), arXiv:1308.0052 [hep-ph].

[25] D. López-Val, T. Plehn, and M. Rauch, JHEP 1310, 134 (2013), arXiv:1308.1979 [hep-ph],

[26] G. Bhattacharyya, D. Das, P. B. Pal, and M. Rebelo, JHEP 1310, 081 (2013), arXiv:1308.4297 [hep-ph].

[27] O. Eberhardt, (2013), arXiv:1309.1278 [hepph],

[28] S. Chang, S. K. Kang, J.-P. Lee, K. Y. Lee, S. C. Park, et al., (2013), arXiv:1310.3374 [hep-ph]. 
[29] A. Celis, V. Ilisie, and A. Pich, JHEP 1312, 095 (2013), arXiv:1310.7941 [hep-ph],

[30] L. Wang and X.-F. Han, arXiv:1312.4759 [hep-ph].

(2013),

[31] O. Eberhardt, U. Nierste, and M. Wiebusch, JHEP 1307, 118 (2013), arXiv:1305.1649 [hep$\mathrm{ph}]$.

[32] K. Cheung, J. S. Lee, and P.-Y. Tseng, JHEP 1401, 085 (2014), arXiv:1310.3937 [hep-ph].

[33] A. Arhrib, P. Ferreira, and R. Santos, (2013), arXiv:1311.1520 [hep-ph].

[34] A. Djouadi, W. Kilian, M. Muhlleitner, and P. Zerwas, Eur.Phys.J. C10, 27 (1999), arXiv:hep-ph/9903229 [hep-ph].

[35] A. Djouadi, W. Kilian, M. Muhlleitner, and P. Zerwas, Eur.Phys.J. C10, 45 (1999), arXiv:hep-ph/9904287 [hep-ph].

[36] U. Baur, T. Plehn, and D. L. Rainwater, Phys.Rev. D69, 053004 (2004), arXiv:hep$\mathrm{ph} / 0310056$ [hep-ph].

[37] M. J. Dolan, C. Englert, and M. Spannowsky, JHEP 1210, 112 (2012), arXiv:1206.5001 [hep$\mathrm{ph}]$.

[38] A. Papaefstathiou, L. L. Yang, and J. Zurita, Phys.Rev. D87, 011301 (2013), arXiv:1209.1489 [hep-ph],

[39] J. Baglio, A. Djouadi, R. Gröber, M. Mühlleitner, J. Quevillon, and M. Spira, JHEP 1304, 151 (2013), arXiv:1212.5581 [hep-ph].

[40] F. Goertz, A. Papaefstathiou, L. L. Yang, and J. Zurita, JHEP 1306, 016 (2013) arXiv:1301.3492 [hep-ph].

[41] A. J. Barr, M. J. Dolan, C. Englert, and M. Spannowsky, (2013), arXiv:1309.6318 [hep$\mathrm{ph}]$.

[42] S. Dawson, S. Dittmaier, and M. Spira, Phys.Rev. D58, 115012 (1998), arXiv:hepph/9805244 [hep-ph].

[43] J. Grigo, J. Hoff, K. Melnikov, and M. Steinhauser, Nucl.Phys. B875, 1 (2013) arXiv:1305.7340 [hep-ph].

[44] D. Y. Shao, C. S. Li, H. T. Li, and J. Wang, JHEP07, 169 (2013), arXiv:1301.1245 [hep$\mathrm{ph}]$.

[45] D. de Florian and J. Mazzitelli, Phys.Lett. B724, 306 (2013), arXiv:1305.5206 [hep-ph].

[46] D. de Florian and J. Mazzitelli, Phys. Rev. Lett. 111, 201801, 201801 (2013), arXiv:1309.6594 [hep-ph].

[47] R. Frederix, S. Frixione, V. Hirschi, F. Maltoni, O. Mattelaer, et al., (2014), arXiv:1401.7340 [hep-ph].

[48] V. Barger, L. L. Everett, C. Jackson, and G. Shaughnessy, (2013), arXiv:1311.2931 [hep- $\mathrm{ph}]$,

[49] A. Arhrib, R. Benbrik, C.-H. Chen, R. Guedes, and R. Santos, JHEP 0908, 035 (2009), arXiv:0906.0387 [hep-ph],

[50] M. J. Dolan, C. Englert, and M. Spannowsky, Phys.Rev. D87, 055002 (2013), arXiv:1210.8166 [hep-ph]

[51] A. Efrati and Y. Nir, (2014), arXiv:1401.0935 [hep-ph].

[52] J. F. Gunion and H. E. Haber, Phys.Rev. D67, 075019 (2003), arXiv:hep-ph/0207010 [hep-ph].

[53] O. Eberhardt, G. Herbert, H. Lacker, A. Lenz, A. Menzel, U. Nierste, and M. Wiebusch, Phys.Rev.Lett. 109, 241802 (2012), arXiv:1209.1101 [hep-ph]

[54] F. Boudjema and A. Semenov, Phys.Rev. D66, 095007 (2002), arXiv:hep-ph/0201219 [hep-ph],

[55] N. G. Deshpande and E. Ma, Phys.Rev. D18, 2574 (1978).

[56] A. Barroso, P. Ferreira, I. Ivanov, and R. Santos, (2013), arXiv:1303.5098 [hep-ph].

[57] I. Ginzburg and I. Ivanov, Phys.Rev. D72, $115010(2005)$.

[58] B. W. Lee, C. Quigg, and H. Thacker, Phys.Rev. D16, 1519 (1977).

[59] H. Huffel and G. Pocsik, Z.Phys. C8, 13 (1981).

[60] J. Maalampi, J. Sirkka, and I. Vilja, Phys.Lett. B265, 371 (1991).

[61] S. Kanemura, T. Kubota, and E. Takasugi, Phys.Lett. B313, 155 (1993), arXiv:hep$\mathrm{ph} / 9303263$ [hep-ph].

[62] A. G. Akeroyd, A. Arhrib, and E.-M. Naimi, Phys.Lett. B490, 119 (2000), arXiv:hep$\mathrm{ph} / 0006035$ [hep-ph].

[63] L. Durand, P. N. Maher, and K. Riesselmann, Phys.Rev. D48, 1084 (1993), arXiv:hep$\mathrm{ph} / 9303234$ [hep-ph].

[64] U. Nierste and K. Riesselmann, Phys.Rev. D53, 6638 (1996), arXiv:hep-ph/9511407 [hep-ph].

[65] CMS physics analysis summary, CMS-PASHIG-13-005 ().

[66] S. Chatrchyan et al. (CMS Collaboration), Eur.Phys.J. C73, 2469 (2013), arXiv:1304.0213 [hep-ex],

[67] CMS physics analysis summary, CMS-PASHIG-13-021 ().

[68] S. Schael et al. (ALEPH, DELPHI, L3, OPAL, SLD, LEP Electroweak Working Group, SLD Electroweak Group, SLD Heavy Flavour Group), Phys.Rept. 427, 257 (2006), arXiv:hepex/0509008 [hep-ex].

[69] R. Harlander, M. Mhlleitner, J. Rathsman, M. Spira, and O. Stl, (2013), arXiv:1312.5571 [hep-ph] 
[70] A. Freitas and Y.-C. Huang, JHEP 1208, 050 (2012), errata ibid. 05(2013)074,10(2013)044, arXiv:1205.0299v3 [hep-ph],

[71] T. Hahn and M. Perez-Victoria, Comput. Phys. Commun. 118, 153 (1999), arXiv:hep$\mathrm{ph} / 9807565$.

[72] T. Hahn, Comput. Phys. Commun. 140, 418 (2001), arXiv:hep-ph/0012260.

[73] T. Hahn and M. Rauch, Nucl. Phys. Proc. Suppl. 157, 236 (2006), arXiv:hep-ph/0601248.

[74] D. Y. Bardin, M. S. Bilenky, T. Riemann, M. Sachwitz, and H. Vogt, Comput.Phys.Commun. 59, 303 (1990).

[75] D. Y. Bardin, P. Christova, M. Jack, L. Kalinovskaya, A. Olchevski, et al., Comput.Phys.Commun. 133, 229 (2001), arXiv:hepph/9908433 [hep-ph].

[76] A. Arbuzov, M. Awramik, M. Czakon, A. Freitas, M. Grunewald, et al., Comput.Phys.Commun. 174, 728 (2006), arXiv:hep$\mathrm{ph} / 0507146$ [hep-ph].

[77] P. Gonzalez, J. Rohrwild, and M. Wiebusch, Eur.Phys.J. C72, 2007 (2012), arXiv:1105.3434 [hep-ph]

[78] M. Spira, (1995), arXiv:hep-ph/9510347 [hep$\mathrm{ph}]$.
[79] A. Djouadi, J. Kalinowski, and M. Spira, Comput.Phys.Commun. 108, 56 (1998), arXiv:hepph/9704448 [hep-ph].

[80] J. Butterworth, F. Maltoni, F. Moortgat, P. Richardson, S. Schumann, et al., (2010), arXiv:1003.1643 [hep-ph].

[81] A. Djouadi, M. Muhlleitner, and M. Spira, Acta Phys.Polon. B38, 635 (2007), arXiv:hepph/0609292 [hep-ph].

[82] M. Wiebusch, Comput.Phys.Commun. 184, 2438 (2012), arXiv:1207.1446 [hep-ph].

[83] publicly available at http://dvegas . hepforge.org/

[84] N. Kauer and D. Zeppenfeld, Phys.Rev. D65, 014021 (2002), arXiv:hep-ph/0107181 [hep-ph],

[85] N. Kauer, Phys.Rev. D67, 054013 (2003). arXiv:hep-ph/0212091 [hep-ph].

[86] A. Hocker, H. Lacker, S. Laplace, and F. Le Diberder, Eur.Phys.J. C21, 225 (2001) arXiv:hep-ph/0104062 [hep-ph].

[87] B. Dumont, J. F. Gunion, Y. Jiang, and S. Kraml, (2014), arXiv:1405.3584 [hep-ph]. 